\title{
Evaluation of Movement Restriction Zone Sizes in Controlling Classical Swine Fever Outbreaks
}

\author{
Shankar Yadav'*, Nicole Olynk Widmar', Donald C. Lay Jr. ${ }^{3}$, Candace Croney ${ }^{1,4}$ and \\ Hsin-Yi Weng ${ }^{1}$
}

'Department of Comparative Pathobiology, Purdue University, West Lafayette, IN, USA, ${ }^{2}$ Department of Agricultural Economics, Purdue University, West Lafayette, IN, USA, ${ }^{3}$ USDA-ARS Livestock Behavior Research Unit, West Lafayette, IN, USA, ${ }^{4}$ Purdue University Center for Animal Welfare Science, West Lafayette, IN, USA

\section{OPEN ACCESS}

Edited by:

Salome Dür,

University of Bern, Switzerland

Reviewed by:

Michael Ward,

University of Sydney, Australia

Flavie Vial,

Epi-Connect, Sweden

Fernando O. Mardones,

Universidad Nacional Andrés Bello,

Chile

*Correspondence:

Shankar Yadav

vetshankar@gmail.com

Specialty section: This article was submitted to Veterinary Epidemiology and

Economics,

a section of the journal Frontiers in Veterinary Science

Received: 30 August 2016 Accepted: 23 December 2016

Published: 10 January 2017

Citation:

Yadav S, Olynk Widmar N, Lay DC Jr., Croney $\mathrm{C}$ and Weng $\mathrm{H}-\mathrm{Y}$

(2017) Evaluation of Movement Restriction Zone Sizes in Controlling Classical Swine Fever Outbreaks.

Front. Vet. Sci. 3:124.

doi: 10.3389/fvets.2016.00124
The objective of this study was to compare the impacts of movement restriction zone sizes of 3, 5, 9, and $11 \mathrm{~km}$ with that of $7 \mathrm{~km}$ (the recommended zone size in the United States) in controlling a classical swine fever (CSF) outbreak. In addition to zone size, different compliance assumptions and outbreak types (single site and multiple site) were incorporated in the study. Three assumptions of compliance level were simulated: baseline, baseline $\pm 10 \%$, and baseline $\pm 15 \%$. The compliance level was held constant across all zone sizes in the baseline simulation. In the baseline $\pm 10 \%$ and baseline $\pm 15 \%$ simulations, the compliance level was increased for 3 and $5 \mathrm{~km}$ and decreased for 9 and $11 \mathrm{~km}$ from the baseline by the indicated percentages. The compliance level remained constant in all simulations for the 7-km zone size. Four single-site (i.e., with one index premises at the onset of outbreak) and four multiple-site (i.e., with more than one index premises at the onset of outbreak) CSF outbreak scenarios in Indiana were simulated incorporating various zone sizes and compliance assumptions using a stochastic between-premises disease spread model to estimate epidemic duration, percentage of infected, and preemptively culled swine premises. Furthermore, a risk assessment model that incorporated the results from the disease spread model was developed to estimate the number of swine premises under movement restrictions that would experience animal welfare outcomes of overcrowding or feed interruption during a CSF outbreak in Indiana. Compared with the 7-km zone size, the 3-km zone size resulted in a longer median epidemic duration, larger percentages of infected premises, and preemptively culled premises ( $P$ 's $<0.001$ ) across all compliance assumptions and outbreak types. With the assumption of a higher compliance level, the $5-\mathrm{km}$ zone size significantly $(P<0.001)$ reduced the epidemic duration and percentage of swine premises that would experience animal welfare outcomes in both outbreak types, whereas assumption of a lower compliance level for 9 - and $11-\mathrm{km}$ zone sizes significantly $(P<0.001)$ increased the epidemic duration and percentage of swine premises with animal welfare outcomes compared with the $7-\mathrm{km}$ zone size. The magnitude of impact due to a zone size varied across the outbreak types (single site and multiple site). Overall, the 7-km zone size was found to be most effective in controlling CSF outbreaks, whereas the 5-km zone size was comparable to the $7-\mathrm{km}$ zone size in some circumstances.

Keywords: classical swine fever, CSF, movement restriction, outbreak control, foreign animal diseases, pig, swine 


\section{INTRODUCTION}

Classical swine fever (CSF) is one of the most economically devastating diseases affecting the swine industry and is currently listed as a Class A foreign animal disease in the United States (1). CSF was first identified in the United States in 1833 and was eradicated in 1976 (2). Currently, CSF is present in Asia, Eastern Europe, and South America. CSF emerged in several disease-free countries and caused severe consequences in the past decades (3). For example, in 1997-1998, severe CSF outbreaks were reported from the Netherlands, Germany, Italy, and Spain. In the Netherlands alone, 11 million pigs were culled and $\$ 2.3$ billion were spent during the outbreak control (4). In 2001-2002, CSF outbreaks in Catalonia, Spain, led to slaughter of 0.29 million pigs (5).

Movement restriction is the most essential strategy for CSF outbreak control (6-8). In the United States, movement restrictions will be implemented and enforced on infected, suspected, and contact swine premises in the infected zone (a 3-km radius surrounding the infected premises) and on the uninfected swine premises in the movement restriction zone ( $7 \mathrm{~km}$ away from the perimeter of the infected zone; see Figure 1A) during a CSF outbreak (9). In the movement restriction zone, movement restrictions will be enforced until 28 days after the disinfection of the last infected premises (9). During this period, the unauthorized movements of pigs, vehicles, and swine farm workers beyond the designated movement restriction areas will be prohibited (9). For the effective implementation of movement restriction,

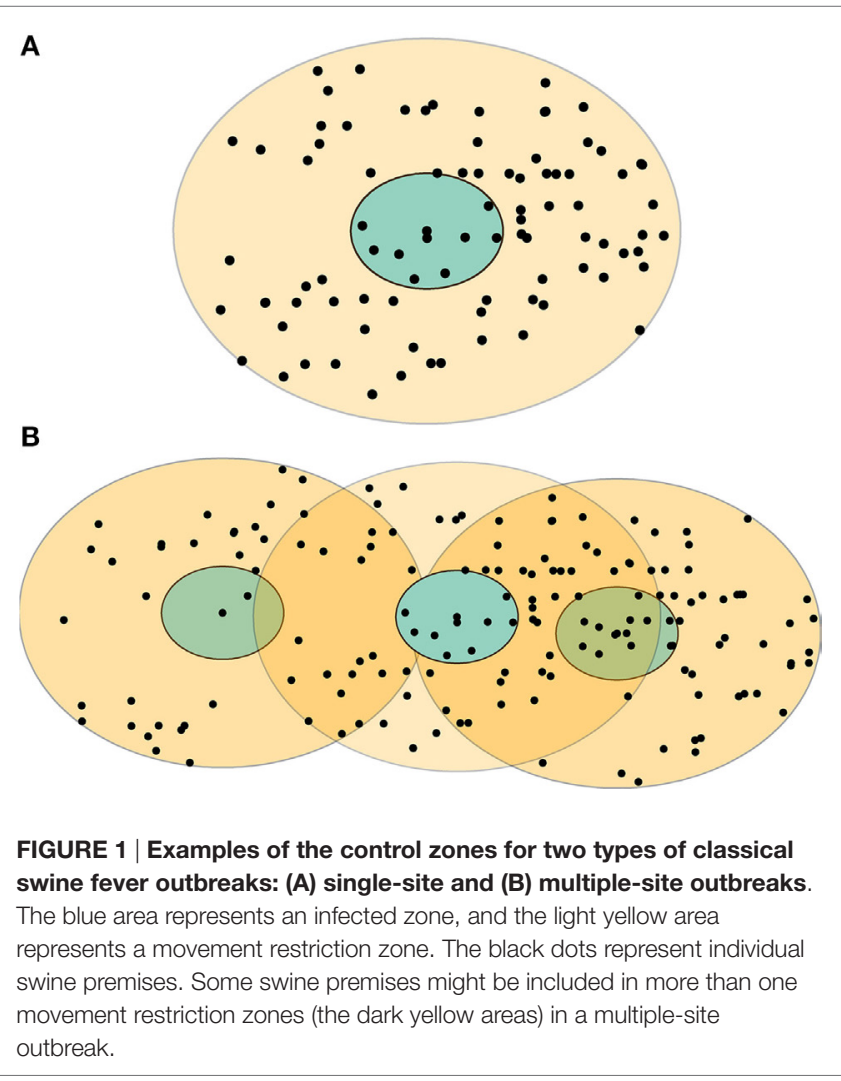

selection of suitable movement restriction zone size could be vital, and it might necessitate consideration of various aspects, such as the initial outbreak types (single-index premises versus multiple-index premises), compliance levels, available resources, and the consequences (e.g., adverse animal welfare outcomes) of movement restriction.

A high level of compliance with movement restriction is essential for effective management of CSF outbreaks (6). However, several factors might contribute to the maintenance of a high compliance level with movement restrictions. For example, compliance level might be dependent on the movement restriction zone size, the progress of an ongoing outbreak, or the initial outbreak types. A smaller movement restriction zone size would require less enforcement to maintain the same level of compliance. A lower compliance with movement restrictions might be observed during the early stages of an epidemic due to lack of proper communication among stakeholders. On the other hand, a prolonged epidemic might result in a lower compliance level toward the later stage of an outbreak. In the past, a maximum of $85 \%$ compliance with movement restriction was estimated during the 1997-1998 CSF outbreak in the Netherlands (8).

Despite its crucial role in CSF outbreak control, various complications might arise due to implementation of movement restriction. For example, US swine herds are operated utilizing the maximum available spaces, and movement restriction might give rise to overcrowding in the swine herds $(10,11)$. Also, movement restriction on feed carrying vehicles might cause feed supply interruptions in swine herds $(10,12)$. These consequences could induce animal welfare problems among the pigs $(10,11)$. In overcrowded swine herds, pigs cannot express their natural behaviors (e.g., exploration or chewing an object), which might result in tail-biting, ear-chewing, aggression, fighting, and wounds $(13,14)$. During a CSF outbreak, the extent and magnitude of such animal welfare outcomes in the affected areas might be dependent on the movement restriction zone size. Historical CSF control programs encountered insufficient resources for effective management due to the large number of depopulated swine premises that resulted from infection, preemptive culling, and animal welfare problems, which complicated the outbreak management and extended the outbreak duration $(15,16)$.

Compared with other CSF control strategies (e.g., preemptive culling and vaccination), movement restriction has been reported to be the most effective in controlling CSF outbreaks (6). However, only limited studies directly evaluated the impacts of movement restriction zone sizes on different CSF outbreak-related outcomes. During the 1997-1998 CSF outbreak in the Netherlands, movement restrictions were inconsistently imposed, and the zone size was extended while the epidemic was at its peak (8). Such inconsistencies in the implementation of movement restriction zone sizes could negatively affect outbreak control efforts. Most of the CSF simulation studies have incorporated a fixed movement restriction zone size (17-20). Choices of movement restriction zone size in previous studies were different than the recommended movement restriction zone size in the United States. Moreover, none of the previous studies evaluated the impacts of movement restriction zone sizes on animal welfare concerns of pigs under movement restriction. Therefore, we conducted this study in the 
context of the Indiana swine industry to evaluate the impacts of the selected movement restriction zone sizes $(3,5,9$, and $11 \mathrm{~km})$ on CSF outbreak-related outcomes [e.g., epidemic duration (ED), percentage of infected premises, preemptively culled premises, and premises affected by adverse animal welfare outcomes] compared with 7-km zone size, which is the recommended zone size in the US CSF outbreak emergency response plan (9).

Indiana is the top fifth pork producing state in the United States. In 2012, there were 8,631 registered swine premises with herd size ranging from 1 to 20,000 . The Indiana swine industry imported 2.8 million and exported 1.5 million live pigs in 2012. The majority of the swine premises were finisher operations (59\%), followed by farrow-to-finish (36\%) and nursery (5\%) operations.

\section{MATERIALS AND METHODS}

In this study, the Indiana State Swine Premises Identification Database (USAHERDS) of 2012 was used. The USAHERDS contained information on individual swine premises and live pig import and export activities. Each individual swine premise was identified by a unique swine premises identification number, geolocation (latitude and longitude), herd size, and operation type. In a previous study, we developed risk metrics to identify the most-likely CSF outbreak scenarios in Indiana (21). We randomly selected 4 (of 19) single-site (i.e., 1 initial outbreak site) and 4 (of 15) multiple-site (i.e., more than 1 initial outbreak sites) CSF outbreak scenarios for outbreak simulations in this study (21). The four multiple-site outbreak scenarios had 26, 20, 17, and 4 initial outbreak sites (i.e., index premises).

ArcGIS (version 10.3, ESRI, Redlands, CA, USA) was used to map the infected zone ( $3-\mathrm{km}$ radius) and movement restriction zones with the sizes of 3, 5, 7, 9, and $11 \mathrm{~km}$ (away from the perimeter of the infected zone) for each of the index premises $(n=71)$ in the eight CSF outbreak scenarios. An example of infected and movement restriction zones and the distribution of swine premises within these control zones in the single-site and multiple-site CSF outbreak types are present in Figures 1A,B, respectively.

A two-step modeling approach was employed in the study. First, CSF spread was simulated using the North American Animal Disease Spread Model (NAADSM) to estimate the impacts of movement restriction zone sizes on $\mathrm{ED}$, percentage of infected, and preemptively culled premises. Second, a novel animal welfare risk assessment model was developed to estimate the percentage of swine premises that would experience animal welfare outcomes of overcrowding and feed interruption under different movement restriction zone sizes.

\section{Simulation of CSF Spread}

North American Animal Disease Spread Model software PC version 4.0.13 was used to simulate CSF spread in Indiana. NAADSM is an open-source software for simulations of infectious disease outbreaks and their control measures. The NAADSM integrates stochastic, temporal, and spatial modeling characteristics (22). For CSF spread simulation, two main data sources were used: empirical data (i.e., USAHERDS) and published literature. Geolocation (latitude and longitude), herd size, and operation type of Indiana swine premises in 2012 were used to simulate the spatial spread of CSF among swine premises. Data on virus transmission were obtained from the published literature (Table 1).

All Indiana swine premises $(N=8,631)$ registered in the 2012 USAHERDS were included in the model simulations. At the start of simulations (day 0), all swine premises were labeled as susceptible status except for the index premises, which were labeled as latently infected status. The latently infected premises were allowed to transit to subclinical and clinical disease status. The duration of different stages of CSF infection (i.e., latent, subclinical, and clinical) and the corresponding probability distributions were obtained from published studies $(6,20,23)$ (Table 1). The transmission of viruses from the infected premises to susceptible premises was allowed through three modes: direct contact, indirect contact, and local spread. A direct contact was defined as the spread of CSF from infected premises to susceptible premises through shipment of live pigs, and indirect contact was through movement of vehicles, people, and equipment. A local spread was defined as the spread of CSF from infected premises to susceptible premises within a $1-\mathrm{km}$ radius $(22,27,28)$. For the spread of viruses via direct or indirect contact, contact rates, probability of infection transfer, and distance distribution of recipient premises were specified. The input parameters for contact rates and probability of disease transfer were adopted from published studies (20, $24,26)$. The NAADSM used the geolocation of swine premises in Indiana to compute the between-premises distance matrix. The probability of disease transfer was modeled to gradually decrease as the distance from an infected premises increased $(22,28)$. Three CSF outbreak control strategies to be implemented in the United States (1) were incorporated in the model simultaneously: movement restriction; vaccination; and depopulation of vaccinated, infected, and contact premises. Vaccination (live attenuated) was implemented in the model for all swine premises in the infected zone $(3-\mathrm{km}$ radius) $(1,9,29)$. The depopulation capacity was modeled to gradually increase from 0 to 10 premises/day by day

\section{TABLE 1 | Premises-level model input parameters and probability} distributions used for the simulation of classical swine fever spread in Indiana, United States.

\begin{tabular}{|c|c|c|}
\hline Parameter & $\begin{array}{l}\text { Probability } \\
\text { distribution }\end{array}$ & Reference \\
\hline Latent period (days) & Poisson (4) & (6) \\
\hline Subclinical period (days) & Poisson (6) & (23) \\
\hline Clinical period (days) & Poisson (21) & (20) \\
\hline $\begin{array}{l}\text { Mean direct contact rate } \\
\text { (recipient premises/premises/day) }\end{array}$ & Poisson (0.186) & (24) \\
\hline $\begin{array}{l}\text { Probability of infection transfer after direct } \\
\text { contact }\end{array}$ & 0.277 & $(25)$ \\
\hline $\begin{array}{l}\text { Mean indirect contact rate } \\
\text { (recipient premises/premises/day) }\end{array}$ & Poisson (0.3) & (26) \\
\hline $\begin{array}{l}\text { Probability of infection transfer after indirect } \\
\text { contact }\end{array}$ & 0.048 & (25) \\
\hline $\begin{array}{l}\text { Maximum distance for contact between } \\
\text { premises }\end{array}$ & $\begin{array}{l}\text { Triangle } \\
(1,60,120)\end{array}$ & USAHERDS ${ }^{a}$ \\
\hline Between-premises distance matrix & $\begin{array}{l}\text { Computed from } \\
\text { data }\end{array}$ & USAHERDS ${ }^{a}$ \\
\hline
\end{tabular}

a2012 Indiana Swine Premises Identification Database. 
7 and 15 premises/day by 15 days onward (24). Additional details on the model parameters and model descriptions can be found elsewhere $(21,22,28)$.

Three different compliance assumptions were simulated: baseline, baseline $\pm 10 \%$, and baseline $\pm 15 \%$. Movement restriction zone sizes were dichotomized into two groups: small ( 3 and $5 \mathrm{~km}$ ) and large $(9$ and $11 \mathrm{~km})$. In the baseline, compliance level was held constant across all zone sizes. In the baseline $\pm 10 \%$, the compliance level was increased by $10 \%$ from the baseline for the small zone size group and decreased by $10 \%$ for the large group. In the baseline $\pm 15 \%$, the compliance level was increased by $15 \%$ from the baseline for the small group and decreased by $15 \%$ for the large group. The compliance level for $7-\mathrm{km}$ zone size remained constant in all simulations. The compliance level was modeled by altering direct contact (e.g., movement of pigs) and indirect contact (e.g., movement of vehicles and people) rates in the NAADSM. For example, a direct contact rate reduced to $25 \%$ reflected a compliance level of no movement of pigs of $75 \%$, and an indirect contact rate reduced to $35 \%$ reflected a compliance level of no movement of people and vehicles of $65 \%$. The baseline compliance levels, modeled as direct and indirect contact rates, by different outbreak types are summarized in Table 2 . We incorporated slightly lower compliance levels for multiple-site outbreak type compared with single-site outbreak assuming that a larger number of swine premises and broader geographical areas in the multiplesite outbreak type would reduce compliance due to a greater demand of resources for enforcement. Indirect contacts were assumed to have a lower compliance than direct contacts due to potentially low adherence of people and vehicles with movement restriction. All simulations were run separately for each of the five movement restriction zone sizes, two outbreak types (with eight outbreak scenarios), and three compliance levels with 500 iterations each. Previous studies have demonstrated that this number of iterations was sufficient to generate reliable output estimates $(22,24)$.

\section{Animal Welfare Risk Assessment Model}

A novel stochastic risk assessment model was developed to estimate the percentage of swine premises that would experience adverse animal welfare outcomes due to movement restrictions

TABLE 2 | Baseline compliance level with movement restrictions expressed as the percent reduction in the direct and indirect contact rates used in the classical swine fever spread model by two types of outbreaks (i.e., single-site outbreak and multiple-site outbreak).

\begin{tabular}{lccccc}
\hline Day & \multicolumn{4}{c}{ Compliance levels } \\
\cline { 2 - 4 } & \multicolumn{2}{c}{ Single-site outbreak } & & Multiple-site outbreak \\
\cline { 2 - 4 } \cline { 5 - 6 } & $\begin{array}{c}\text { Direct contact } \\
\text { rate }\end{array}$ & $\begin{array}{c}\text { Indirect contact } \\
\text { rate }\end{array}$ & & $\begin{array}{c}\text { Direct contact } \\
\text { rate }\end{array}$ & $\begin{array}{c}\text { Indirect contact } \\
\text { rate }\end{array}$ \\
\hline 1 & 0 & 0 & & 0 & 0 \\
7 & $75 \%$ & $65 \%$ & & $70 \%$ & $65 \%$ \\
15 & $75 \%$ & $65 \%$ & & $70 \%$ & $65 \%$ \\
30 & $75 \%$ & $60 \%$ & & $70 \%$ & $60 \%$ \\
60 & $65 \%$ & $60 \%$ & $60 \%$ & $60 \%$
\end{tabular}

in the context of the Indiana swine industry. The animal welfare outcomes investigated were overcrowding or feed interruption on the finisher swine operations, which were the major swine operations in Indiana in 2012. The unit for the model simulation was swine premises and simulations proceeded by a time step of one day. The probability distribution of input model parameters such as the unique number of swine premises that would fall under movement restrictions during an outbreak was estimated using the 2012 Indiana swine premises data. The estimation of probability distribution for ED was described in this section, whereas the time elapsed between the onset of an outbreak and the emergence of animal welfare concerns (TWC) was adopted from our previous study (10). Details on the input model parameters and their probability distributions are summarized in Table 3 and are briefly described below.

ArcGIS (version 10.3, ESRI, Redlands, CA, USA) was used to map the infected zones and movement restriction zones for each of the index premises at the first time step of the eight mostlikely CSF outbreak scenarios. The premises that were withinmovement restriction zones were identified and counted. Two linear regression equations were developed based on these data to estimate the number of swine premises that would fall under movement restrictions during an outbreak (Table 3). As shown in Figure 1B, some premises might fall under multiple movement restriction zones (i.e., overlap) in a multiple-site outbreak. The percentage of overlapped swine premises was estimated for a given number of infected premises to approximate the number of unique swine premises. If the overlap percentage was greater than or equal to a predetermined maximum overlap percentage, the maximum percentage of overlap (maximum overlap, in Table 3) was used. The ED was estimated from the CSF spread model described in Section "Simulation of CSF Spread." Unlike multiple-site outbreak type, the ED estimates from the single-site outbreak type had a bimodal distribution (denoted by ED low and ED high in Table 3). The probability distribution of ED in the single-site outbreak scenario was represented by two trianglular (minimum, most likely, maximum) distributions along with a Bernoulli (probability of event) distribution, which represented the probability of occurrence of either of the ED (low or high) distributions (Table 3).

Adverse animal welfare consequences of movement restriction investigated in this study included overcrowding and feed interruption. When the total weight of pigs on premises exceeded $100-115 \%$ of the maximum capacity of the premises, the condition was referred to as overcrowding. The maximum capacity of swine premises was calculated as the total weight of pigs on premises at the age of harvest. Feed interruption was modeled as a farmer's decision to discontinue feed supply, followed by the euthanasia of the pigs. The decision of feed discontinuity was dependent on the ED estimated at the onset of an outbreak, the duration between the initial age of the pigs at the start of an outbreak and the harvest age, and the progress (number of days) of an outbreak; longer durations of these factors resulted in the farmers' being more likely to discontinue the feed supply. We developed a model to estimate the time when overcrowding or feed interruption emerged (TWC) in our previous study (10). Probability distributions for TWC were derived from those estimates. 
TABLE 3 | Input model parameters and probability distributions (estimated using the 2012 Indiana swine premises data) for animal welfare risk assessment models by movement restriction (MR) zone size.

\begin{tabular}{|c|c|c|c|c|c|}
\hline \multirow[t]{2}{*}{ Parameters } & \multicolumn{5}{|c|}{ Movement restriction zone sizes } \\
\hline & 3 km & $5 \mathrm{~km}$ & $7 \mathrm{~km}$ & 9 km & 11 km \\
\hline \multicolumn{6}{|l|}{ Epidemic duration (ED) } \\
\hline Single site: ED low & Triangle $(0,30,130)$ & Triangle $(0,30,130)$ & Triangle $(0,30,130)$ & Triangle $(0,30,130)$ & Triangle $(0,30,130)$ \\
\hline Single site: ED high & Triangle $(210,290,450)$ & Triangle $(170,250,470)$ & Triangle $(170,260,490)$ & Triangle $(130,250,550)$ & Triangle $(130,250,600)$ \\
\hline Single site: Prob_ED_low & Bernoulli $(0.27)$ & Bernoulli $(0.28)$ & Bernoulli (0.28) & Bernoulli (0.3) & Bernoulli (0.3) \\
\hline Multiple site & Triangle $(229,258,316)$ & Triangle $(186,222,313)$ & Triangle $(171,214,315)$ & Triangle $(164,214,337)$ & Triangle $(161,215,336)$ \\
\hline \multicolumn{6}{|l|}{$\begin{array}{l}\text { Time to animal welfare } \\
\text { concern (TWC) }\end{array}$} \\
\hline Single site & Triangle $(2,62,165)$ & Triangle $(2,62,165)$ & Triangle $(2,62,165)$ & Triangle $(2,62,165)$ & Triangle $(2,62,165)$ \\
\hline Multiple site & Triangle $(2,61,173)$ & Triangle $(2,61,173)$ & Triangle $(2,61,173)$ & Triangle $(2,61,173)$ & Triangle $(2,61,173)$ \\
\hline $\begin{array}{l}\text { Number of premises in MR } \\
\text { given number of infected } \\
\text { premises (regression slope) }\end{array}$ & Normal (10.91, 0.186) & Normal $(17.75,1.15)$ & Normal $(24.83,1.93)$ & Normal $(35.82,2.17)$ & Normal $(42.5,3.16)$ \\
\hline $\begin{array}{l}\text { \% overlap given number } \\
\text { of infected premises } \\
\text { (regression slope) }\end{array}$ & Normal $(0.0175,0.0009)$ & Normal $(0.0213,0.0009)$ & Normal $(0.023,0.001)$ & Normal $(0.0224,0.002)$ & Normal $(0.0267,0.002)$ \\
\hline $\begin{array}{l}\text { \% overlap given number } \\
\text { of infected premises } \\
\text { (regression intercept) }\end{array}$ & Normal (-0.0244, 0.012) & Normal (-0.0297, 0.015) & Normal $(-0.0174,0.015)$ & Normal $(-0.005,0.028)$ & Normal $(0.002,0.033)$ \\
\hline Maximum overlap & Triangle $(0.75,0.85,0.95)$ & Triangle $(0.75,0.85,0.95)$ & Triangle $(0.75,0.85,0.95)$ & Triangle $(0.75,0.85,0.95)$ & Triangle $(0.75,0.85,0.95)$ \\
\hline
\end{tabular}

The number of daily newly infected swine premises was estimated from the CSF spread model using the NAADSM (see Simulation of CSF Spread). The number of unique swine premises under movement restrictions was then estimated as a function of the number of infected premises using the regression equations (Table 3). The model algorithms compared TWC with ED to flag when TWC $<\mathrm{ED}$, indicating that the premises would experience adverse animal welfare outcomes before the outbreak ended. A total of 26 different simulations were run using @Risk software (Palisade Corporation, Ithaca, NY, USA) with 100,000 iterations each. Latin hypercube sampling with a Mersenne Twister generator of randomly selected initial seed was used.

\section{Sensitivity Analysis}

For the CSF spread model, direct contact rate and probability of CSF transmission were chosen for the sensitivity analyses because of their major roles in disease spread and also the inconsistent estimates across studies $(18,20,26,30)$. A $25 \%$ change in the values of these parameters was used in the analyses to evaluate their influences on the estimates of ED and the percentage of infected premises. A parameter was considered influential if the median ED changed by 14 days or the median percentage of infected premises changed by $25 \%$ compared with the baseline.

For the animal welfare risk assessment model, the Spearman correlation was performed on all input parameters to assess their correlation with the outcome (i.e., the number of premises that would experience animal welfare outcomes). The parameters that resulted in a correlation coefficient $\geq 0.3$ were included in the further sensitivity analysis. A 25\% change in the values of the selected parameters was used in the analyses to evaluate their influences on the estimates of the median percentage of premises that would experience animal welfare outcomes. An input parameter was considered as influential if the outcome changed by $25 \%$ compared with the baseline.

\section{Statistical Analysis}

Kruskal-Wallis tests were used to compare among the movement restriction zone sizes. Dunn's tests, adjusted for multiple comparisons, were used to compare between $7-\mathrm{km}$ zone size and the other zone sizes after a significant Kruskal-Wallis test. Statistical significance is defined as $P \leq 0.05$.

\section{RESULTS}

A total of 71 infected zones and movement restriction zones were identified and mapped for the zone sizes of 3,5, 7, 9, and $11 \mathrm{~km}$ in the 8 selected CSF outbreak scenarios. The median number of swine premises in the infected zone $(3-\mathrm{km}$ radius of index premises) at the onset of the outbreak was 6 (range: 5-16) and 94 (range: 16-145) in the single-site and multiple-site outbreak type, respectively. Details of the number of unique swine premises in the various movement restriction zone sizes at the onset of an outbreak are presented in Table 4.

\section{Epidemic Duration}

The estimates of ED resulting from different movement restriction zone sizes are presented in Figure 2. In the baseline compliance simulations, 3- and 5-km zone sizes resulted in a significantly 
TABLE 4 | Median (range) number of swine premises in the movement restriction zone sizes of $3,5,7,9$, and $11 \mathrm{~km}$ at the onset of single-site and multiple-site CSF outbreak scenarios in Indiana.

\begin{tabular}{lccccc}
\hline Outbreak & \multicolumn{5}{c}{ Movement restriction zone sizes } \\
\cline { 2 - 6 } scenarios & $\mathbf{3 ~ k m}$ & $\mathbf{5} \mathbf{~ k m}$ & $\mathbf{7} \mathbf{~ k m}$ & $\mathbf{9 ~ k m}$ & $\mathbf{1 1} \mathbf{~ k m}$ \\
\hline Single site & 22 & 42 & 67 & 93 & 122 \\
& $(10-49)$ & $(16-75)$ & $(26-96)$ & $(58-132)$ & $(67-174)$ \\
Multiple site & 207 & 353 & 521 & 735 & 876 \\
& $(45-280)$ & $(75-428)$ & $(137-559)$ & $(205-824)$ & $(268-979)$
\end{tabular}

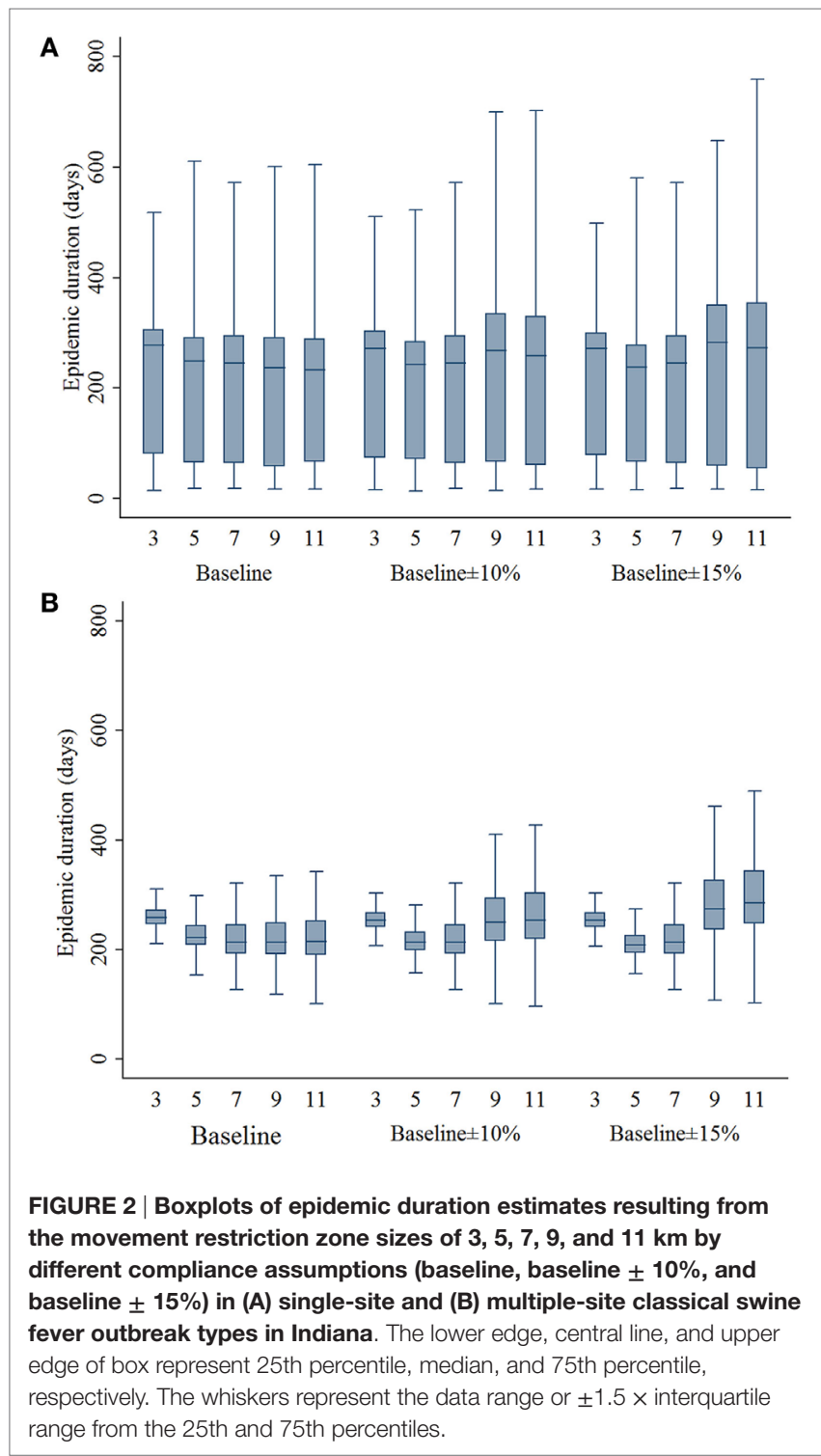

longer ED compared with the 7-km zone size in both outbreak types ( $P$ 's $<0.001)$. In the baseline $\pm 10 \%$ simulations, EDs resulting from 3-, 9-, and 11-km zone sizes were longer compared with the 7-km zone size ( $P$ 's $<0.001)$, whereas the EDs resulting from 5 - and 7-km zone sizes were not different in both outbreak types. In the baseline $\pm 15 \%$ simulations, 3-, 9-, and 11-km zone sizes

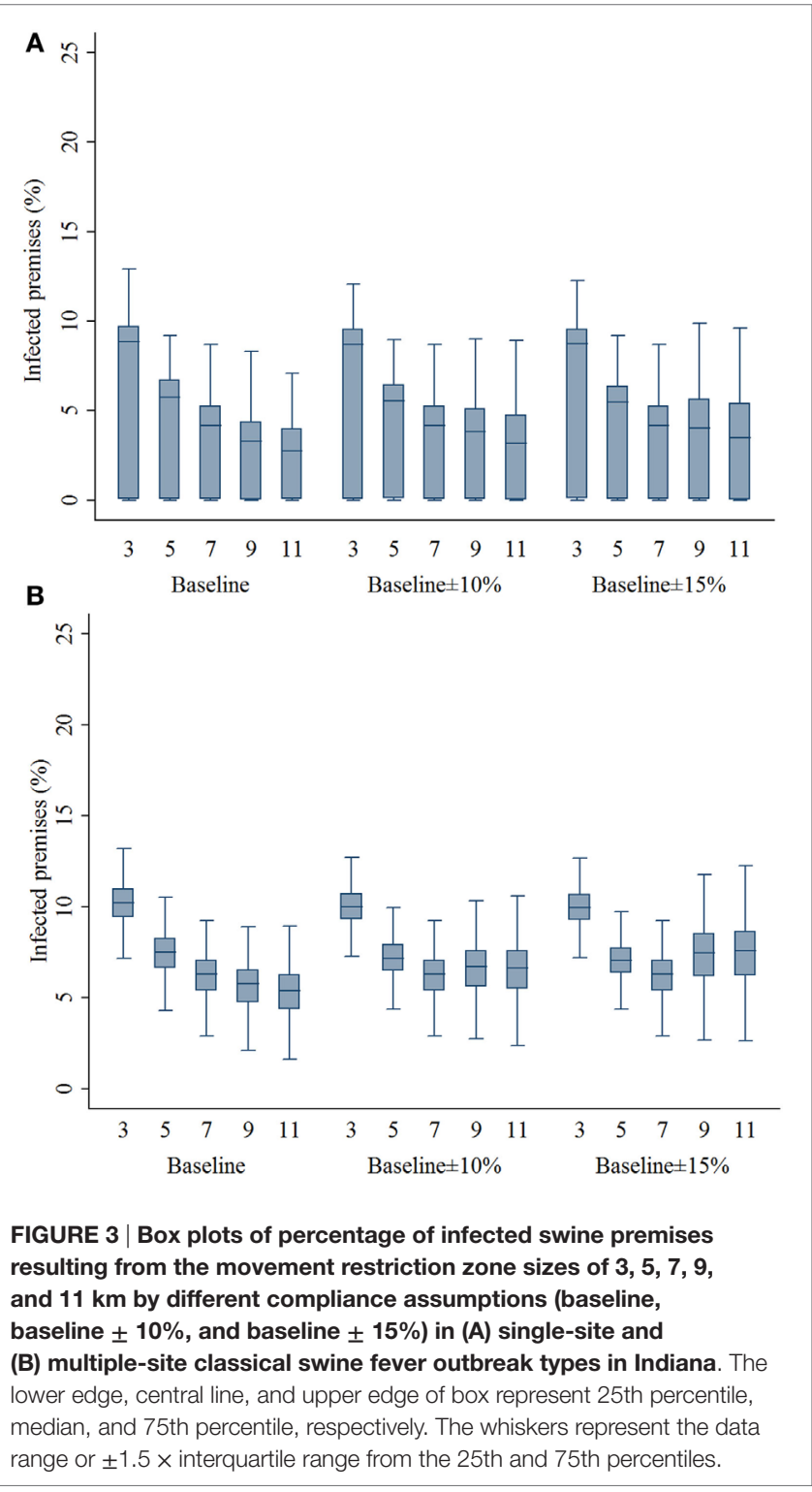

resulted in a significantly longer ED ( $P$ 's $<0.001)$, whereas the $5-\mathrm{km}$ zone size resulted in a shorter ED $(P=0.036)$ compared with the $7-\mathrm{km}$ zone size.

\section{Infected and Preemptively Depopulated Premises}

Compared with the $7-\mathrm{km}$ zone size, the 3 - and $5-\mathrm{km}$ zone sizes resulted in a higher percentage of infected swine premises in both outbreak types ( $P$ 's $<0.001$; Figures 3 A,B $)$. With the assumptions of lower compliance levels, the zone sizes of 9 and 11 resulted in a higher percentage of infected premises in the multiple-site outbreak significantly $(P$ 's $<0.001)$. In the single-site outbreak, the percentage of infected premises resulted from 9-km zone size was not different from that of $7-\mathrm{km}$ zone size $(P=0.537)$. Results of the percentage of infected premises are presented in Figure 3. As with the infected premises estimates, a similar pattern was 
found in the percentage of preemptively culled swine premises (Figure 4).

\section{Adverse Animal Welfare Outcomes}

The percentage of swine premises that would experience adverse animal welfare outcomes due to movement restriction was positively associated with the zone size regardless of compliance levels and outbreak types (Figure 5). The median percentage of swine premises that would experience adverse animal welfare outcomes was $10-27 \%$ lower comparing $3-$ and $5-\mathrm{km}$ zone sizes with $7-\mathrm{km}$ zone size $(P$ 's $<0.001)$.

\section{Composition of Depopulated Swine Premises}

The results of composition of depopulation (due to infection, preemptive culling, and animal welfare outcomes) are presented

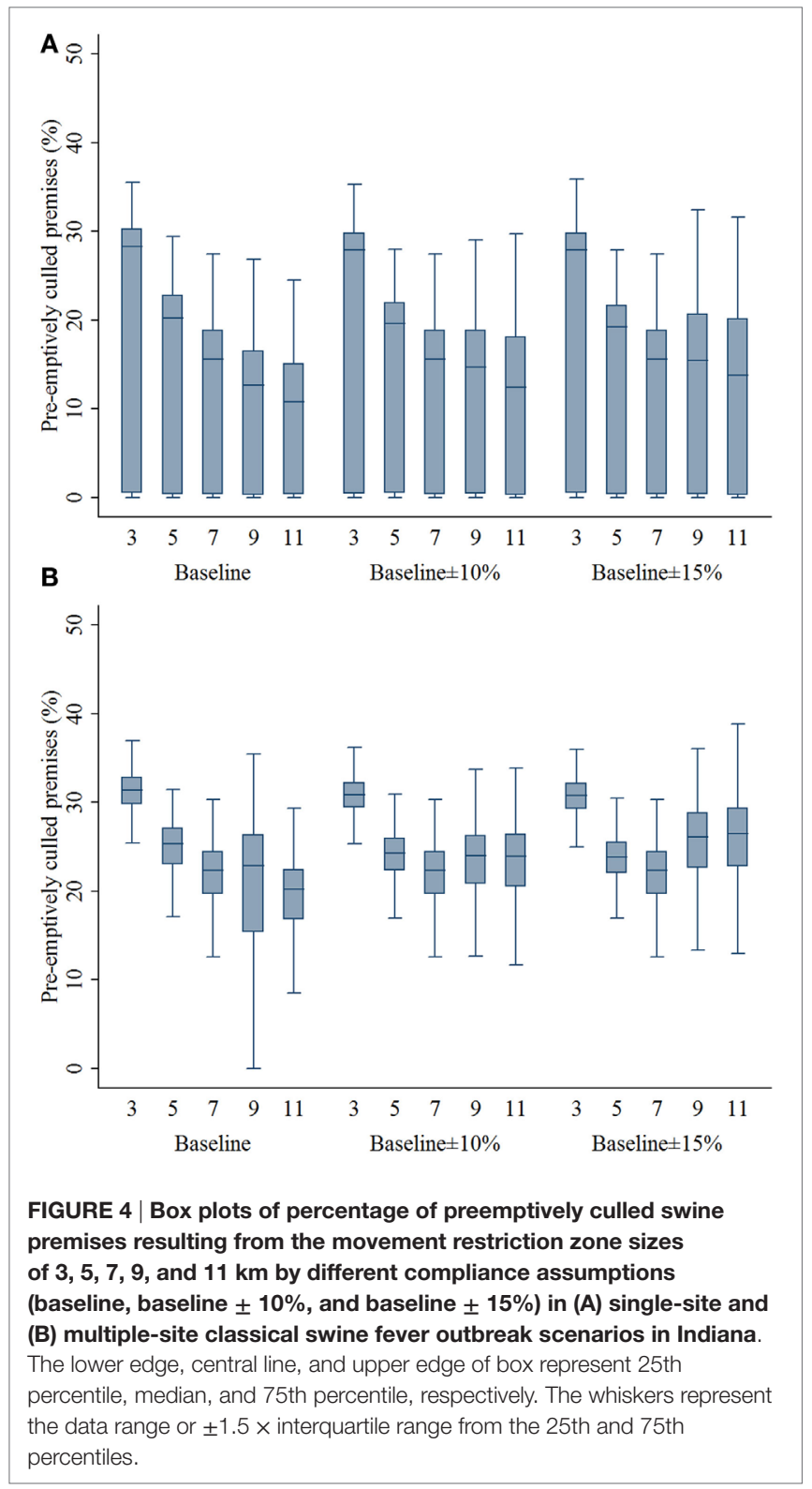

in Figure 6. In an event of CSF outbreak in the United States, infected and contact premises and premises experiencing animal welfare outcomes are all subject to depopulation. Among the small zone sizes ( 3 and $5 \mathrm{~km}$ ), majority of the swine premises to be depopulated were due to preemptive culling (single-site outbreak: 56\%, multiple-site outbreak: $49 \%$ ), whereas among the large zone sizes ( 9 and $11 \mathrm{~km}$ ), the majority to be depopulated were due to animal welfare outcomes (single-site outbreak: 51\%, multiple-site outbreak: 55\%). For the $7-\mathrm{km}$ zone size, 48 and $42 \%$ of the swine premises were preemptively depopulated and 39 and $47 \%$ were depopulated due to animal welfare outcomes in the single-site and multiple-site outbreak, respectively.

\section{Sensitivity Analysis}

The results of the sensitivity analysis for CSF spread model showed that direct contact rate and probability of infection transfer were

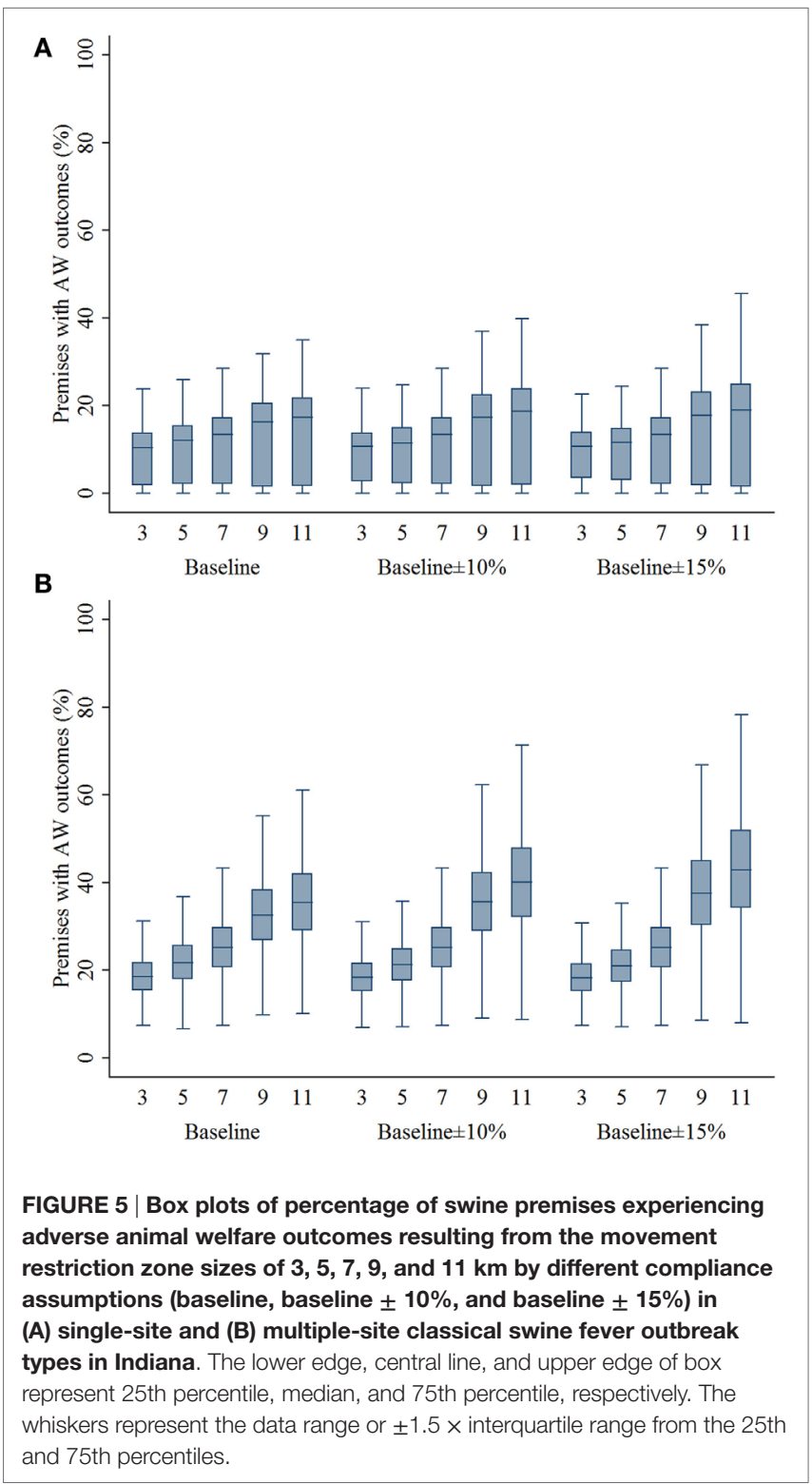



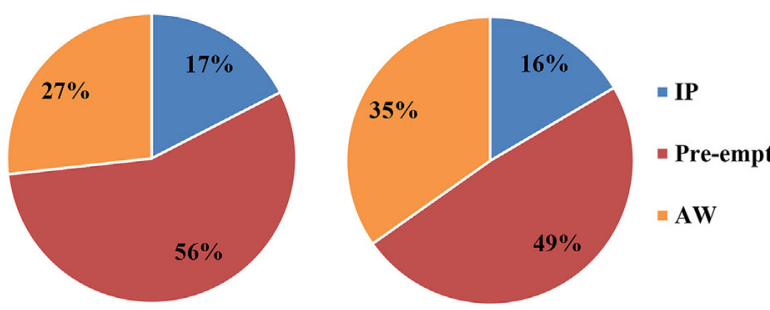

Single-site (zone size $<7-\mathrm{km}$ )
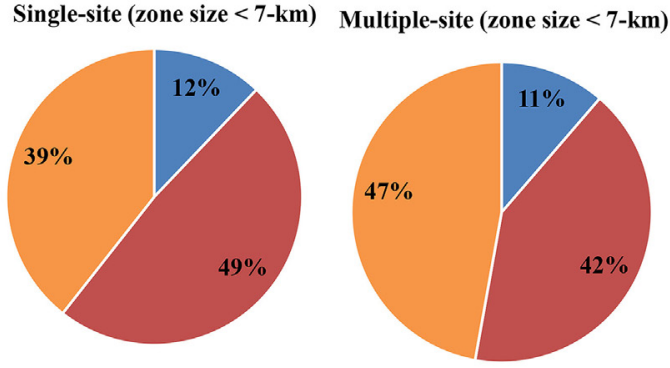

Single-site (zone size $=7-\mathrm{km})$

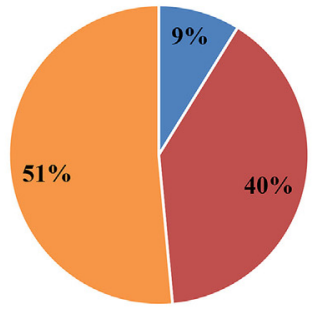

Multiple-site (zone size $=7-\mathrm{km})$

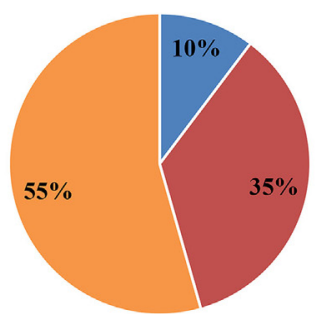

Single-site (zone size $>7-\mathrm{km})$

Multiple-site (zone size > 7-km)

FIGURE 6 | Pie chart represents the percentage of total depopulation of swine premises due to infection (IP), pre-emptive culling (Preempt), and adverse animal welfare outcomes (AW) for small zone sizes ( 3 and $5 \mathrm{~km}$ ), 7-km zone size, and big zone sizes ( 9 and $11 \mathrm{~km}$ ) by outbreak types (single site and multiple site).

influential on the percentage of infected premises estimations in the single-site outbreak. In the multiple-site outbreak, a $25 \%$ increase in direct contact rate or probability of infection transfer altered the estimate of median percentage of infected premises by $26-65 \%$ across different zone sizes. They showed a greater influence on the percentage of infected premises estimate for 3-, 9-, and 11-km zone sizes.

Direct contact rate and probability of infection transfer were also influential on ED estimations for all zone sizes in the multiple-site outbreak. In the single-site outbreak, a 25\% increase in direct contact rate and probability of infection transfer altered the ED estimate by $22-45$ days.

For the animal welfare risk assessment model, maximum overlap percentage (maximum overlap; Table 3 ) and probability of ED low (Prob_ED_low; Table 3) showed a correlation with the number of premises experiencing animal welfare outcome estimate (i.e., Spearman's correlation coefficient $\geq 0.3$ ) and were included in the further sensitivity analysis. The results of the sensitivity analysis showed that a change in maximum overlap by 0.05 altered the outcome estimate up to $27 \%$ across different zone sizes in the multiple-site outbreak. In the single-site outbreak, a decrease in the maximum overlap by 0.05 did not affect the outcome estimate, whereas an increase by 0.05 reduced the number of affected swine premises by $29-41 \%$ across the investigated zone sizes. Prob_ED_low was changed to Bernoulli (0.1) and Bernoulli (0.4) from the baseline distributions in the sensitivity analysis. These changes in Prob_ED_low did not influence the median percentage of swine premises that experienced animal welfare outcomes estimate.

\section{DISCUSSION}

We evaluated the impact of various movement restriction zone sizes on CSF outbreak control compared with the recommended zone size of $7 \mathrm{~km}$ using Indiana swine premises data. The study findings provide evidence-based foundation for decision makers to determine the optimal movement restriction zone size for CSF outbreak controls.

The investigated movement restriction zone sizes showed variable effects on the ED estimates depending on the outbreak types (e.g., single-site and multiple-site outbreaks) and compliance levels. Across the outbreak types, the differences in ED estimates among zone sizes were slightly greater in multiple-site outbreak than in single-site outbreak. The results also suggested that ED did not always decrease with an increase in the zone size. This was contradictory to the presumption of the monotonically negative relationship between movement restriction zone size and $\operatorname{ED}(6,31)$.

Similarly, it was found that the relationship between movement restriction zone size and the percentage of swine premises to be depopulated (infected premises, preemptively culled premises, and premises with animal welfare outcomes) was not necessarily directional. Both compliance level and outbreak type influenced the effects of movement restriction zone size on the percentage of swine premises to be depopulated. However, the $3-\mathrm{km}$ zone size consistently increased the number of total depopulated swine premises across all simulated scenarios. A closer look at the simulation results revealed that a $3-\mathrm{km}$ zone size resulted in the largest number of daily new infections compared with other zone sizes. With a constant compliance level (i.e., baseline compliance simulation), 5-, 9-, and 11-km zone sizes resulted in the similar percentage of premises to be depopulated. Assumption of a low compliance level for a larger zone size led to a higher percentage of depopulation for zone sizes of 9 and $11 \mathrm{~km}$; a greater difference was found in the multiple-site outbreak compared with single-site outbreak. The finding that the differences in outcome estimates tended to be lager in the multiple-site outbreak might be explained by that a multiple-site outbreak, on average, resulted in more new infections. Overall, 7-km zone size was found to result in the lowest percentage of premises to be depopulated, whereas the $5-\mathrm{km}$ zone size showed comparable results in the baseline compliance simulation and performed better with the assumption of a higher compliance level in the multiple site. Having fewer premises that need to be depopulated during an outbreak may greatly reduce the overall burden (e.g., resources for euthanasia, carcass disposal, and transport) for outbreak controls $(32,33)$. During the 1997-1998 CSF outbreaks in the Netherlands, seven million pigs were euthanized to alleviate adverse animal welfare outcomes, 
which competed for the limited resources going toward euthanizing infected pigs, carcass disposal, biosecurity, and disinfection. Consequently, the epidemic was prolonged, which might have caused additional animal welfare issues in pigs and economic losses $(4,34)$. A similar challenge of insufficient resources for euthanasia, carcass transport, and disposal was observed in the highly pathogenic avian influenza outbreaks in the United States in 2014, which also hindered the timely management of outbreaks (35).

Our models showed that preemptive culling and euthanasia due to adverse animal welfare outcomes were the major contributors to the total depopulation, whereas the infected premises contributed the least (Figure 6). This finding agrees with the reports from historical CSF outbreaks (3). Our results further indicated that the composition of depopulation was dependent on movement restrictions zone size. In the small zone size group, the majority of swine premises were euthanized due to preemptive culling, whereas in the large zone size group, adverse animal welfare outcomes contributed the most. In the historical outbreaks, euthanasia of pigs due to adverse animal welfare outcomes contributed $64 \%$ to total pigs euthanized $(6,8)$. The study model showed that movement restriction zone size of $5 \mathrm{~km}$ could result in $20 \%$ fewer premises to be euthanized due to adverse animal welfare outcomes compared with the zone size of $11 \mathrm{~km}$. These findings underscore the significance of animal welfare problems if a bigger movement restriction zone size is designated. Our assessment of different outbreak-related outcomes provide more comprehensive evidence to assist decision makers and disease control authorities in designating an optimal movement restriction zone size for CSF outbreak control. Awareness of swine producers toward these crucial outcomes might also help them be better prepared to prevent devastating consequences in an event of CSF outbreak.

Overall, the 7-km movement restriction zone size had the best performance in CSF outbreak control based on our models. It resulted in the shortest ED and the lowest percentage of swine premises to be depopulated compared with the other investigated zone sizes. A shorter ED will reduce the international trade ban period, which will lead to a quicker recovery of business. Furthermore, implementing a $7-\mathrm{km}$ zone size may reduce the demand of limited resources for outbreak controls, which is crucial for smooth and efficient outbreak management. Under certain circumstances, such as in an event of multiple-site CSF outbreak, a $5-\mathrm{km}$ zone size might be as effective as a $7-\mathrm{km}$ zone size in controlling the outbreak in Indiana; particularly if a higher compliance level can be achieved.

\section{REFERENCES}

1. USDA. APHIS Foreign Animal Disease Framework Response Strategies: FAD Preparedness and Response Plan Manual 2-0. Riverdale, MD: Animal and Plant Health Inspection Service, Veterinary Services (2012).

2. Penrith ML, Vosloo W, Mather C. Classical swine fever (hog cholera): review of aspects relevant to control. Transbound Emerg Dis (2011) 58(3):187-96. doi:10.1111/j.1865-1682.2011.01205.x

3. Edwards S, Fukusho A, Lefevre PC, Lipowski A, Pejsak Z, Roehe P, et al. Classical swine fever: the global situation. Vet Microbiol (2000) 73(2-3):10319. doi:10.1016/S0378-1135(00)00138-3
Movement restrictions will inevitably also affect regular movements of pigs outside the designated control zones. Further investigation by including the movement data outside the control zones is warranted to more comprehensively evaluate the impacts of movement restriction zone size. During a CSF outbreak, the interstate network of swine movement might result in a rapid spread of CSF beyond Indiana. Therefore, including the other partnering swine producing states of Indiana in future studies might help achieve better insight about the role of movement restriction zone size in controlling a multistate CSF outbreak. The extrapolation of the study findings to the swine industry of other US states and countries should be done with due consideration of the assumptions in the model. The results and simulation outputs from this study could be used in the cost-benefit analysis of movement restriction in controlling a CSF outbreak.

\section{CONCLUSION}

The effectiveness of movement restriction zone size in controlling a CSF outbreak were dependent on various factors, such as outbreak type, compliance with movement restriction, and outcome measure of interest. Our findings indicated that a $7-\mathrm{km}$ zone size was the most effective in reducing the ED and percentage of swine premises that need to be depopulated compared with other investigated zone sizes. The zone size of $5 \mathrm{~km}$ was comparable with the 7-km zone size particularly in the multiple-site outbreak with an assumption of a higher compliance level.

\section{AUTHOR CONTRIBUTIONS}

SY: conceived and designed the study, performed data collection, simulation, data analysis, and manuscript writing. NOW: contributed in data analysis, manuscript writing, and review. DL: contributed in manuscript writing and review. CC: contributed in manuscript writing and review. H-YW: contributed in study design, data analysis, manuscript writing, and review.

\section{ACKNOWLEDGMENTS}

The authors thank Dr. Marianne Ash, Dr. Maria Cooper, and the Indiana State Board of Animal Health for sharing with us the selected information on farm locations in Indiana. The work is supported by the USDA National Institute of Food and Agriculture, Hatch project 230919 and is partially funded by the National Pork Board (Project \# 13-153).

4. Stegeman A, Elbers A, Smit HD, Moser H, Smak J, Pluimers F. The 1997-1998 epidemic of classical swine fever in the Netherlands. Vet Microbiol (2000) 73(2-3):183-96. doi:10.1016/S0378-1135(00) 00144-9

5. Allepuz A, Casal J, Pujols J, Jove R, Selga I, Porcar J, et al. Descriptive Epidemiology of the Outbreak of Classical Swine Fever in Catalonia (Spain). London: British Veterinary Association (2001).

6. Thulke HH, Eisinger D, Beer M. The role of movement restrictions and pre-emptive destruction in the emergency control strategy against CSF outbreaks in domestic pigs. Prev Vet Med (2011) 99(1):28-37. doi:10.1016/ j.prevetmed.2011.01.002 
7. Velthuis A, Mourits M. Effectiveness of movement-prevention regulations to reduce the spread of foot-and-mouth disease in the Netherlands. Prev Vet Med (2007) 82(3):262-81. doi:10.1016/j.prevetmed.2007.05.023

8. Terpstra C, de Smit AJ. The 1997/1998 epizootic of swine fever in the Netherlands: control strategies under a non-vaccination regimen. Vet Microbiol (2000) 77(1-2):3-15. doi:10.1016/S0378-1135(00)00252-2

9. USDA. Classical Swine Fever Response Plan: The Red Book. Riverdale, MD: Animal and Plant Health Inspection Service, Veterinary Services (2013). Available from: https://www.aphis.usda.gov/animal_health/emergency_management/downloads/csf_responseplan.pdf

10. Weng HY, Yadav S, Olynk Widmar NJ, Croney C, Ash M, Cooper M. Modelling the time at which overcrowding and feed interruption emerge on the swine premises under movement restrictions during a classical swine fever outbreak. Animal (2016) 1-7. doi:10.1017/S1751731116001609

11. Bargen LL, Whiting TL. Time to critical overcrowding of Manitoba swine barns in the event of restriction on animal movement. Can Vet J (2002) 43(11):855-62.

12. Laurence CJ. Animal welfare consequences in England and Wales of the 2001 epidemic of foot and mouth disease. Rev Sci Tech (2002) 21(3):863-8. doi:10.20506/rst.21.3.1382

13. Kittawornrat A, Zimmerman JJ. Toward a better understanding of pig behavior and pig welfare. Anim Health Res Rev (2011) 12(01):25-32. doi:10.1017/ S1466252310000174

14. Keeling LJ. Healthy and happy: animal welfare as an integral part of sustainable agriculture. Ambio (2005) 34(4):316-9. doi:10.1579/0044-7447-34.4.316

15. Elbers AR, Stegeman A, Moser H, Ekker HM, Smak JA, Pluimers FH. The classical swine fever epidemic 1997-1998 in the Netherlands: descriptive epidemiology. Prev Vet Med (1999) 42(3-4):157-84. doi:10.1016/ S0167-5877(99)00074-4

16. Scudamore JM, Harris DM. Control of foot and mouth disease: lessons from the experience of the outbreak in Great Britain in 2001. Rev Sci Tech (2002) 21(3):699-710. doi:10.20506/rst.21.3.1351

17. Mangen MJJ, Burrell AM, Mourits MCM. Epidemiological and economic modelling of classical swine fever: application to the 1997/1998 Dutch epidemic. Agric Syst (2004) 81(1):37-54. doi:10.1016/j.agsy.2003.08.003

18. Boklund A, Toft N, Alban L, Uttenthal A. Comparing the epidemiological and economic effects of control strategies against classical swine fever in Denmark. Prev Vet Med (2009) 90(3-4):180-93. doi:10.1016/j.prevetmed.2009. 04.008

19. Durr S, Dohna HZ, Labio ED, Carpenter TE, Doherr MG. Evaluation of control and surveillance strategies for classical swine fever using a simulation model. Prev Vet Med (2013) 108(1):73-84. doi:10.1016/j.prevetmed.2012.07.006

20. Martinez-Lopez B, Ivorra B, Ramos AM, Sanchez-Vizcaino JM. A novel spatial and stochastic model to evaluate the within-and between-farm transmission of classical swine fever virus. I. General concepts and description of the model. Vet Microbiol (2011) 147(3-4):300-9. doi:10.1016/j.vetmic.2010.07.009

21. Yadav S, Widmar NJO, Weng H-Y. Modeling classical swine fever outbreak-related outcomes. Front Vet Sci (2016) 3:7. doi:10.3389/fvets.2016. 00007

22. NAADSM. North American Animal Disease Spread Model (NAADSM) Development Team. Version 4.0.13. Free Program Distributed via the Internet. (2013). Available from: http://www.naadsm.org

23. Klinkenberg D, Nielen M, Mourits MC, de Jong MC. The effectiveness of classical swine fever surveillance programmes in the Netherlands. Prev Vet Med (2005) 67(1):19-37. doi:10.1016/j.prevetmed.2004.10.003
24. McReynolds SW, Sanderson MW, Reeves A, Hill AE. Modeling the impact of vaccination control strategies on a foot and mouth disease outbreak in the Central United States. Prev Vet Med (2014) 117(3):487-504. doi:10.1016/ j.prevetmed.2014.10.005

25. Mangen MJ, Nielen M, Burrell AM. Simulated effect of pig-population density on epidemic size and choice of control strategy for classical swine fever epidemics in the Netherlands. Prev Vet Med (2002) 56(2):141-63. doi:10.1016/ S0167-5877(02)00155-1

26. Karsten S, Rave G, Krieter J. Monte Carlo simulation of classical swine fever epidemics and control. I. General concepts and description of the model. Vet Microbiol (2005) 108(3-4):187-98. doi:10.1016/j.vetmic.2005.04.009

27. Mintiens K, Laevens H, Dewulf J, Boelaert F, Verloo D, Koenen F. Risk analysis of the spread of classical swine fever virus through "neighbourhood infections" for different regions in Belgium. Prev Vet Med (2003) 60(1):27-36. doi:10.1016/S0167-5877(03)00080-1

28. Reeves A, Hualo R. Calculating Probabilities of Local Area and Airborne Disease Spread in NAADSM 4. Fort Collins, CO: Colorado State University (2012).

29. USDA. APHIS Foot and Mouth Disease Response Plan: The Red Book. Riverdale, MD: Animal and Plant Health Inspection Service, Veterinary Services (2012).

30. Jalvingh AW, Nielen M, Maurice H, Stegeman AJ, Elbers AR, Dijkhuizen AA. Spatial and stochastic simulation to evaluate the impact of events and control measures on the 1997-1998 classical swine fever epidemic in the Netherlands. I. Description of simulation model. Prev Vet Med (1999) 42(3-4):271-95.

31. Nielen M, Jalvingh AW, Meuwissen MP, Horst SH, Dijkhuizen AA. Spatial and stochastic simulation to evaluate the impact of events and control measures on the 1997-1998 classical swine fever epidemic in the Netherlands. II. Comparison of control strategies. Prev Vet Med (1999) 42(3-4):297-317. doi:10.1016/S0167-5877(99)00081-1

32. East I, Roche S, Wicks R, de Witte K, Garner M. Options for managing animal welfare on intensive pig farms confined by movement restrictions during an outbreak of foot and mouth disease. Prev Vet Med (2014) 117(3):533-41. doi:10.1016/j.prevetmed.2014.10.002

33. Roche S, Garner M, Wicks R, East I, de Witte K. How do resources influence control measures during a simulated outbreak of foot and mouth disease in Australia? Prev Vet Med (2014) 113(4):436-46. doi:10.1016/ j.prevetmed.2013.12.003

34. Thrusfield M, Mansley L, Dunlop P, Taylor J, Pawson A, Stringer L. The foot-and-mouth disease epidemic in Dumfries and Galloway, 2001. 1: characteristics and control. Vet Rec (2005) 156(8):229-52. doi:10.1136/vr.156.9.269

35. USDA. Fall 2015 Highly Pathogenic Avian Influenza (HPAI) Preparedness and Response Plan. Washington, DC: United States Department of Agriculture (USDA), Animal and Plant Health Inspection Services (APHIS), Veterinary Services (2015).

Conflict of Interest Statement: The authors declare that the research was conducted in the absence of any commercial or financial relationships that could be construed as a potential conflict of interest.

Copyright $\odot 2017$ Yadav, Olynk Widmar, Lay, Croney and Weng. This is an open-access article distributed under the terms of the Creative Commons Attribution License (CC BY). The use, distribution or reproduction in other forums is permitted, provided the original author(s) or licensor are credited and that the original publication in this journal is cited, in accordance with accepted academic practice. No use, distribution or reproduction is permitted which does not comply with these terms. 